\title{
Robot-Assisted and Device-Based Rehabilitation of the Upper Extremity
}

\section{(ㄷ) (1) (우)}

Autoren

Michael Sailer ${ }^{1,2}$, Catherine M. Sweeney-Reed ${ }^{3}$, Juliane Lamprecht ${ }^{2}$

Institute

1 MEDIAN Neurological Rehabilitation Center Magdeburg \& MEDIAN Rehabilitation Center Flechtingen

2 Institute for Neurorehabilitation affiliated to Ottovon-Guericke University, Magdeburg

3 Neurocybernetics and Rehabilitation, Clinic for Neurology and Neurosurgery, Otto-von-Guericke University, Magdeburg

Key words

robot-assisted, device-based rehabilitation, upper extremity, robotics, neurorehabilitation

Bibliography

DOI https://doi.org/10.1055/s-0043-116174

Neurology International Open 2017; 1: E242-E246

(c) Georg Thieme Verlag KG Stuttgart · New York

ISSN 2511-1795

Correspondence

Prof. Dr. med. Michael Sailer

MEDIAN Klinik NRZ Magdeburg

Gustav-Ricker-Straße 4

39120 Magdeburg

michael.sailer@median-kliniken.de

\section{ABSTRACT}

Neurorehabilitation of patients with upper limb motor dysfunction due to central nervous system damage still lacks adequate standardization. During the last decade, robot- and deviceassisted rehabilitation has become more feasible for the treatment of functional disorders of the upper limb after stroke. Here we present an overview of technological aspects and differential use of devices for upper limb rehabilitation as well as a review of relevant clinical studies. We also discuss the potential for standardized evaluation in the context of limited health care resources. The effectiveness of device-assisted therapy, in comparison to conventional approaches, remains a matter of debate, largely due to the heterogeneous design of the available clinical studies. However, we believe that a better understanding of the timing, intensity, and quality of upper limb rehabilitation, as well as technological progress, will lead to the establishment of a central role for robot- and deviceassisted rehabilitation in the next decade.

\section{Introduction}

Improvement of the functionality of the upper limb after an injury to the central nervous system (CNS) is one of the most important tasks of neurorehabilitation. Stroke is the leading cause of upper limb disability, with a range of complex functional upper limb impairments occurring in approximately 50 to 70 percent of cases [1]. In addition, these patients commonly exhibit sensory-motor deficits of the lower extremity, speech impairment, visual defects, and cognitive deficits during the acute phase. Even limited dysfunction of the upper extremity can result in significant limitations of daily activities and quality of life [2]. The probability of regaining sufficient hand function, i. e., grasping adequate for performance of everyday activities, in the presence of a pronounced functional disorder due to a distal paresis or hand paralysis, is at most 20 percent
[3]. Effective therapy of the upper limb is therefore a crucial component of neurorehabilitation.

In recent years, neurorehabilitative therapy for motor deficits has focused on task-specific training, comprising repetitive, context-specific exercises. In addition, introduction of "shaping" exercises at the individual patient's limits of motion, as well as active or passive repetitive activities to reinforce motor learning, should be considered essential foundations of rehabilitative therapy.

A uniform standard of therapy for upper extremity sensorimotor deficits is not currently in place, and individual variation in deficits renders such a standardization unlikely. Based on 109 publications, the guidelines of the German Society for Neurorehabilitation (Deutsche Gesellschaft für Neurorehabilitation), "Rehabilitative Therapy of Arm Paresis after Stroke" published in 
2009 [4] provide recommendations regarding the timing, duration, and intensity of therapy. The highest levels ( $A$ and $B$ ) of recommended therapy contain subgroups of repetitive exercises for gripping and releasing to treat paresis of the hand with partially retained proximal motor function. These include damage-oriented training for arm capacity, basic arm training, constraint-induced movement therapy, mirror therapy, and mental training, as well as neuromuscular electrostimulation (NMES). Robotics-supported upper limb therapy provides a potential adjunct, particularly for those unable to perform the therapeutic motions independently, and is classified as recommendation level B (therapy that should be carried out), i. e., offering average efficacy with a medium to high degree of supporting evidence, based on studies of device-supported therapy focusing on stereotypical movements, without specific task-oriented exercises.

Despite considerable growth in recent years in the number of studies investigating the efficacy of robot-assisted interventions in improving arm function and daily activity performance, the methodological heterogeneity of the studies has led to the conclusion in recent Cochrane meta-analyses that the evidence remains limited $[5,6]$. Nonetheless, a systematic review and meta-analysis this year suggested there may be improvement in motor control and muscle strength [7].

In the next sections, we provide an overview of the current state of technological developments as well as clinical applications.

\section{Technical Developments}

In contrast to the devices employed for lower extremity rehabilitation, which target gait initiation and complex movement of the ankle, knee joint, and hip as one unit, devices developed for the upper extremity can be divided into systems which: a) support the whole arm (shoulder, elbow, forearm, lower arm, wrist, fingers); b) train only partial aspects of upper limb movement; and c) focus solely on repetitive movement of the fingers [8].

Devices for supporting upper limb movement, e.g. as a part of learning to walk again post-stroke, can be classified as using either the end-effector or the exoskeleton approach. In the former, movement is transferred from the device to the distal parts of the upper limb, while the latter provides complete control of movement of the entire limb.

\section{End-effector devices}

End-effector-based devices have distal (finger, hand, or wrist) contact with the extremity. Movement of the end-effector initiates movement of the distal portion of the upper limb, thereby inducing movement and altering the position of proximal segments of the upper limb. There is a mechanically-induced chain of movement from distal to proximal. This motion induced by the end-effector can also indirectly change the position of other parts of the body. The MIT-Manus [9] or the MIME (Mirror Image Motion Enabler) [10], as well as devices for finger movement, such as RehaDigit [11], are prototypes of such end-effector devices for the therapy of a functional disorder of the upper limb.

\section{Exoskeleton devices}

Exoskeleton-based devices transmit direct force to segments of the extremity, inducing and guiding movements of the human skeleton, which results in controlled motion of the corresponding joint of the affected extremity. The extent of the movement to be exercised can be managed by independent control of each joint involved. To avoid injuries, this continuously guided exercise requires a very precise adaptation of the individual segments of the device to the body of the rehabilitant, with constant monitoring of the motions transmitted by the device as well as the positions of the affected extremity. Control becomes increasingly complex with greater degrees of freedom allowed in a joint by the device. Consequently a longer set-up time is required for the initial adaptation than is necessary for the use of end-effector devices. However, modern exoskeletons can store the individual positions of the segments or an individual pattern of the respective patient, thereby reducing the subsequent set-up times. The ArmeoPower is an example of an exoskeleton possessing 7 degrees of freedom, supporting movements in the shoulder (adduction/abduction; flexion/extension; inner/ outer rotation), elbow (flexion/extension), lower arm (pronation/ supination), wrist (flexion/extension), and fingers (gripping function).

The advantage of the end-effector approach is a reduced technical complexity and control compared to an exoskeleton device. The disadvantage lies in reduced regulation of the joints proximal to the force transmission of the end-effector, resulting in less control of the proximally induced movements.

\section{Feedback Mechanisms}

Robot- and device-supported therapy enables the recording and processing of diverse signals to control movement, influence movement intensity, and provide feedback of complex motion to the rehabilitant. These signals can be provided as visual, acoustic, or tactile responses.

In general, three types of signals, can be provided as feedback to the rehabilitant and therapist: 1 ) dynamic signals that reproduce the exerted forces in the joints, 2) kinematic signals indicating the position, orientation, and acceleration in the joint, 3) trigger signals used to initiate a movement, including a starting signal and threshold signals related to certain conditions, such as achieving a target set externally by the therapist. These are often used in gaming programs or in a virtual reality environment to increase motivation [12]. These signals may be categorized as high- or low-level control signals. It has been postulated that high-level control signals in particular promote neuroplasticity. Such signals include complete support of the intended movement (assistive control) or the motivation of/challenge to the rehabilitant to perform the intended movement as independently as possible (challenge-based control). On the other hand, signals used exclusively for measurement or reproduction of e. g., force or position, without the active, supporting and guiding function of a device, are considered part of a low-level control strategy [13].

Brain-computer interfaces $(\mathrm{BCl})$ are increasingly being employed to enable patients to use their brain electrical activity to con- 
trol the timing of the visual and proprioceptive feedback they receive from robotic devices as well as from NMES [14]. It has been proposed that temporally coupling brain processes with movement induction enhances motor learning through mechanisms involving neural plasticity.

\section{Clinical Practice}

Initial studies of robot-assisted therapy in patients with severe arm plegia or paresis, were conducted more than 20 years ago, employing the MIT-Manus and MIME devices. The MIT-Manus focuses on elbow and shoulder motion using a planar movement. The MIME device likewise provides only up to two degrees of freedom, also involving a planar movement in the elbow and the shoulder. Despite the limited degrees of freedom in movement, the use of both end-effector devices has resulted in an increase both in proximal arm force and in the range of active proximal arm movement $[9,10]$. It can be assumed that repetition of the exercise and/or its intensity contributed to the functional improvement. It must, however, be taken into account that in such studies, high intensity neurorehabilitative interventions are used, independent of the method. For example, positive effects on the functional capability of the upper limb have also been identified in studies investigating non-robotic but device-based therapies (devices for guiding upper limb movement without active mechanical support), as well as in studies of structured, intensive, conventional therapies (standardized repetitive practice and shaping) $[15,16]$. Sensorimotor Active Rehabilitation Training (SMART) is an example of an established and non-robot-supported procedure. Arm stretching and bending is practiced by fixation on the wrist and guidance of motion on a rail, thus reducing the degrees of freedom. In 12 interventions using the SMART method for one hour three times a week, arm function (strength and extent of arm stretching) improved compared to the control group not using SMART procedures. The disadvantage of this procedure is that the rehabilitant must have adequate upper limb function, thereby excluding patients with severe arm paresis [15]. Training with the Bi-Manu-Track uses a similar approach, also improving arm strength and selective arm mobility. Unlike the SMART procedure, bi-manual movement is performed either by passive or active movements of the forearm (pro-/supination) and the wrist (flexion/extension) by the unaffected arm, and/or actively against resistance provided by the device [17].

For example, significant improvement of selective hand mobility, as measured using the Fugl-Meyer test, was demonstrated treating a plegic hand using a mechanical finger trainer (RehaDigit) during a four-week therapy phase with 20 min of training 5 days a week [11]. The mechanism performed a passive, repetitive movement of the fingers (excluding the thumb). On the other hand, a multi-center study of 127 patients with exclusively chronic impairment of the upper limb (on average 4.9 years after stroke) did not demonstrate a greater improvement of arm function in a devicesupported therapy cohort compared with a patient cohort undergoing conventional intensive therapy [16]. However, an advantage was shown in the device-supported therapy group after 36 weeks in comparison to standard therapy [16].

A robot-based exoskeleton device potentially provides optimal guidance, with active support of the entire movement of the im-

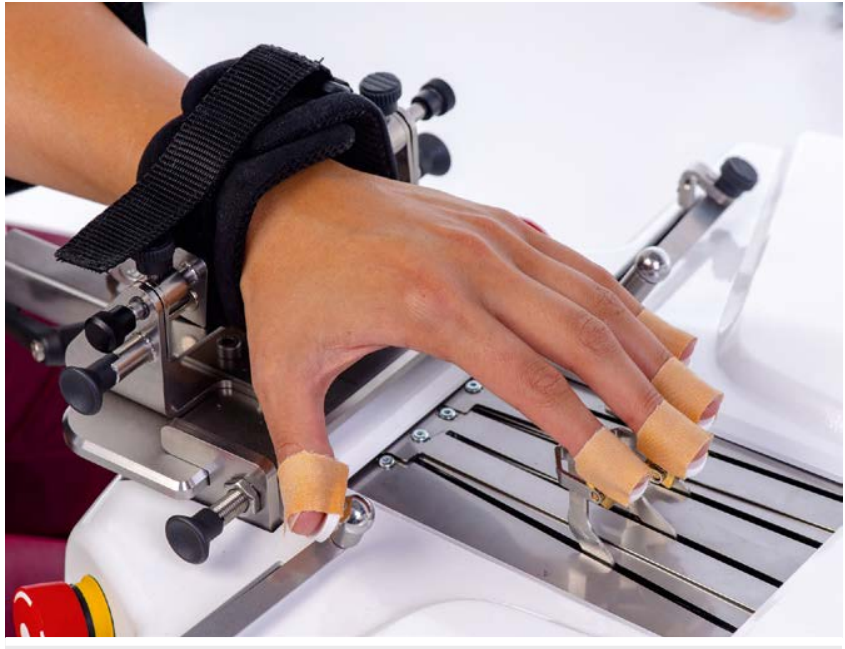

- Fig. 1 Amadeo. This device is an end-effector using an electric drive that via fixation of the wrist supports the position and force of each finger, including the thumb.

paired limb. A recent pilot study using a system actively supporting upper extremity movement, including the shoulder, elbow, flexion and extension in the wrist, and the gripping function, showed robot-assisted intervention led to a significantly greater improvement in upper limb function (Action Research Arm Test) than comparable intensive conventional therapy [18]. Moreover, improvement in the Box and Blocks Test was greater in conventional therapy if it followed robot-supported therapy in the cross-over design. A further study of robot-based hand therapy, in which the robot provided compensation for finger movement deficit, demonstrated improvement in the Fugl-Meyer and Box and Block Test [19]. The Amadeo end-effector system ( $\triangleright$ Fig. 1) was used in a randomized, controlled study $(\mathrm{N}=20)$ and compared with intensive ergotherapy after a 4-week intervention ( $5 x$ per week). However, interpretation of the reported improvement must take into account the limited number of cases.

\section{Further Developments and Summary}

The greatest barrier to early motor rehabilitation using targeted therapy is non-existent movement, such that patients are unable to perform a specific, task-oriented exercise, underlining the need for novel approaches for this group [3].

Further development and utilization of robot- and device-supported therapy of the upper extremity can usefully supplement conventional therapy. However, the effectiveness of device-supported therapy of the upper limb, compared to equally intensive standard therapy, is still debatable.

With regard to the cost-effectiveness of device-assisted therapy, the expense of conventional therapy with equivalent intensity must be considered. A direct expense comparison of both types of therapy has revealed little difference [16]. However, to date no studies have focused on cost-effectiveness.

Most device-based therapies are designed not only to maximize the repetition of training sessions, but also to affect the patient's motivation and attention, optimizing effort levels. The diverse ap- 


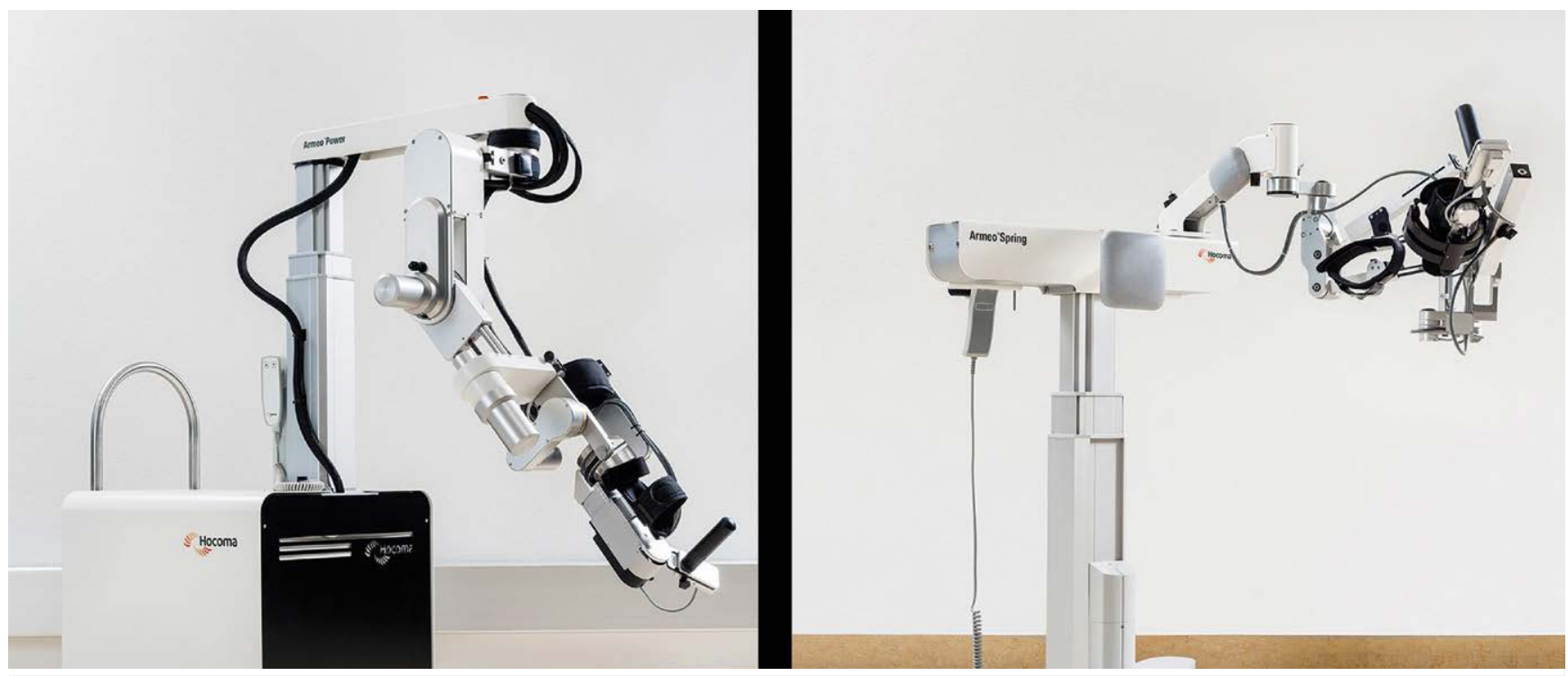

- Fig. 2 ArmeoPower and ArmeoSpring. The ArmeoPower system (Hocoma, left) uses pneumatic drives to perform movements of the upper extremity, including the shoulder (exoskeleton). In comparison, the ArmeoSpring system (Hocoma, right) is a resistance system requiring activity of the rehabilitant's affected limb. Guidance of the movement is provided in the distal region; in contrast, the shoulder does not receive proximal guidance but is included in the chain of movements.

proaches to improving outcome underline the importance of promoting a uniform language for robot- and device-supported therapy in order to better compare and interpret the results of studies. Moreover, the diverse disciplines involved in rehabilitation, ranging from clinical specialties to biomedical engineers and neuroscientists, necessitates the development of common terminologies to facilitate bridging between clinical needs and technological advances [20]. Due to the growing body of technical developments and related data pertaining to upper extremity rehabilitation, it will be necessary to distinguish between device types (end-effector/exoskeleton), as well as to consider optimal support, compensatory guidance, the affected extremity, including the degrees of freedom, and the quality of feedback.

In addition to arm strength and range of movement as outcomes, the focus should also include reliable data reflecting activities of daily living (ADL), reflected in the body function and activity categories of the ICF (International Classification of Functioning). Meta-analyses have confirmed that in addition to the methodological heterogeneity of the studies, robot-supported movements do not comprehensively exercise the complex actions generally required to perform daily activities $[5,7]$. Furthermore, the mechanisms underlying motor learning, e. g., timing of the different regeneration phases, are still largely unexplained.

In this context, everyday training situations need to be conceived and established to extend the previous range of movement repetitions. A combination of robot-based and Constraint-Induced Movement Therapy could also be promising, as recently demonstrated in an RCT study with 34 chronic stroke patients [21].

Although the intensity of the therapy plays a role [4], the optimal duration and frequency of device-based intervention has yet to be established. The additional therapies required by the patient may be decisive. A treatment plan may be usefully based on the functional deficit or on the patient's rehabilitative process, using devices such as Amadeo, ArmeoPower, ArmeoSpring ( $\triangleright$ Fig. 1,2), Pablo, or Tyrostation. In this way, several stations can be connected to other end-effector or exoskeleton devices to provide circuit training or other single or group therapies such as mirror therapy.

Organizational requirements for infrastructure planning have a significant influence on the continuous utilization of the equipment. These include, for example, rooms, staffing, required apparatus, and management of patient transport. Also important organizationally is the integration of robot and device-supported therapy into the therapeutic concept as well as the development of concrete courses of treatment.

Last but not least, the hygiene requirements for the devices and exercise procedure must be considered in the context of multiresistant organisms (MRE). By defining a hygiene plan for device disinfection, combined with procedural instructions for disinfection between patients and, for example, patient-specific belt systems, patients with specific MRE colonization can also be effectively treated using robot- or device-supported therapy.

Robotic and device-based therapy makes a significant contribution to ensuring sufficient movement repetitions with differentiated feedback mechanisms, while allowing therapists to focus on the motivational aspects of rehabilitation. The important role of the interaction between patient and therapist, that comprises both the expertise and the encouragement of active engagement, is a major factor for compliance and the success of rehabilitation [22]. Particularly for patients with pronounced paresis or a plegia, the therapist can take a role in supervising and motivating therapy, rather than being responsible for the dynamic execution of the movement itself. Contact between the rehabilitant and therapist and his/her expertise is and will remain a core component of rehabilitation. With the inclusion of robot-based therapy in neurorehabilitation, further education of therapists and physicians active in rehabilitation focused on motor learning must also be intensified so 
that the potential of this therapy can be optimally utilized and further developed.

Robot- and device-supported therapy represents a potentially useful addition to intensive, task-specific conventional therapy for motor deficits of the upper limb.

\section{Conflict of Interest}

The authors declare no conflict of interest.

\section{References}

[1] Persson HC, Parziali M, Danielsson A et al. Outcome and upper extremity function within 72 hours after first occasion of stroke in an unselected population at a stroke unit: A part of the SALGOT study. BMC Neurol 2012; 12: 162

[2] Nichols-Larsen DS, Clark PC, Zeringue A et al. Factors influencing stroke survivors' quality of life during subacute recovery. Stroke 2005; 36: $1480-1484$

[3] Kwakkel G, Kollen B], van der Grond J et al. Probability of regaining dexterity in the flaccid upper limb: Impact of severity of paresis and time since onset in acute stroke. Stroke 2003; 34: 2181-2186

[4] Platz T, Roschka S. Rehabilitative Therapie bei Armparese nach Schlaganfall. Neurol Rehabil 2009; 15: 81-106

[5] Mehrholz J, Pohl M, Platz T et al. Electromechanical and robot-assisted arm training for improving activities of daily living, arm function, and arm muscle strength after stroke (Update). Cochrane Database Syst Rev 2015; CD006876

[6] Mehrholz J, Hädrich A, Platz T et al. Electromechanical and robotassisted arm training for improving generic activities of daily living, arm function, and arm muscle strength after stroke. Cochrane Database Syst Rev 2012; CD006876

[7] Veerbeek JM, Langbroek-Amersfoort AC, van Wegen EEH et al. Effects of robot-assisted therapy for the upper limb after stroke. Neurorehabil Neural Repair 2017; 31: 107-121

[8] Maciejasz P, Eschweiler J, Gerlach-Hahn K et al. A survey on robotic devices for upper limb rehabilitation. J Neuroeng Rehabil 2014; 11: 3

[9] Volpe BT, Krebs HI, Hogan N et al. A novel approach to stroke rehabilitation: Robot-aided sensorimotor stimulation. Neurology 2000; 54: 1938-1944
[10] Lum PS, Burgar CG, Shor PC et al. Robot-assisted movement training compared with conventional therapy techniques for the rehabilitation of upper-limb motor function after stroke. Arch Phys Med Rehabil 2002; 83: 952-959

[11] Hesse S, Kuhlmann $\mathrm{H}$, Wilk J et al. A new electromechanical trainer for sensorimotor rehabilitation of paralysedfingers: A case series in chronic and acute stroke patients. J Neuroeng Rehabil 2008; 5: 21

[12] Goršič M, Cikajlo I, Novak D. Competitive and cooperative arm rehabilitation games played by a patient and unimpaired person. J Neuroeng Rehabil 2017; 14: 23

[13] Marchal-Crespo L, Reinkensmeyer DJ. Review of control strategies for robotic movement training after neurologic injury. J Neuroeng Rehabil 2009; 6: 20

[14] van Dokkum LEH, Ward T, Laffont I. Brain computer interfaces for neurorehabilitation - its current status as a rehabilitation strategy post-stroke. Ann Phys Rehabil Med 2015; 58: 3-8

[15] Barker RN, Brauer SG, Carson RG. Training of reaching in stroke survivors with severe and chronic upper limb paresis using a novel non-robotic device: A randomized clinical trial. Stroke 2008; 39 : 1800-1807

[16] Lo AC, Guarino PD, Richards LG et al. Robot-assisted therapy for long-term upper-limb impairment after stroke. N Engl J Med. 2010; 362: 1772-1783

[17] Hesse S, Werner C, Pohl M et al. Computerized arm training improves the motor control of the severely affected arm after stroke. A single-blinded randomized trial in two centres. Stroke 2005; 36 : 1960-1966

[18] Brokaw EB, Nichols D, Holley RJ et al. Robotic therapy provides a stimulus for upper limb motor recovery after stroke that is complementary to and distinct from conventional therapy. Neurorehabil Neural Repair 2014; 28: 367-376

[19] Sale P, Mazzoleni S, Lombardi V et al. Recovery of hand function with robot-assisted therapy in acute stroke patients: A randomized-controlled trial. Int J Rehabil Res. 2014; 37: 236-242

[20] Shirota C, Jansa J, Diaz J et al. On the assessment of coordination between upper extremities. J Neuroeng Rehabil 2016; 13: 80

[21] Hsieh Y-w, Liing R-j, Lin K-c et al. Sequencing bilateral robot-assisted arm therapy and constraint-induced therapy improves reach to press and trunk kinematics in patients with stroke. J Neuroeng Rehabil 2016; 13: 31

[22] Hall AM, Ferreira PH, Maher CG. The Influence of the therapist-patient relationship on treatment outcome in physical rehabilitation: A systematic review. Phys Ther 2010; 90: 1099-1110 